\title{
Connaissances et savoir-faire déclarés des internes de médecine générale en matière de contraception : une étude menée en subdivision d'internat de Poitou-Charentes (France)
}

\author{
Reported contraception knowledge and skills of graduate students \\ in general medicine: a study conducted between May and November 2011 \\ in Poitou-Charentes (France)
}

Stéphanie GRANDCOLIN ${ }^{1}$, Jean DU BREUILLAC ${ }^{2}$, François BIRAULT ${ }^{2}$, José GOMES ${ }^{2}$ et Anne-Laure CHAPUIS ${ }^{2}$

1 Service de gynécologie-obstétrique, Centre hospitalier universitaire de Poitiers, France

2 Département de médecine générale, Faculté de médecine, Université de Poitiers, France

Manuscrit reçu le 23 mars 2013 ; commentaires éditoriaux formulés aux auteurs le $1^{\mathrm{er}}$ septembre 2013 et le 17 février 2014 ; accepté pour publication le 18 février 2014

\begin{abstract}
Mots-clés contraception ; connaissances ; habiletés ; internes de médecine générale ; besoins de formation

Résumé - Contexte : La contraception est un domaine important dans le cadre du programme que suivent les internes du diplôme d'études spécialisées (DES) de médecine générale. But : Recueillir les connaissances et savoir-faire déclarés des internes du DES de médecine générale en matière de contraception et identifier leurs besoins de formation dans ce domaine. Méthodes : Nous avons interrogé des internes du DES de médecine générale à l'aide d'un questionnaire administré en ligne sur internet. Les items utilisés ont été déterminés à l'issue d'une démarche de consensus formalisé en recourant à un panel d'experts. Résultats : Aucun des dix-sept items de connaissances et de savoir-faire testés n'était significativement mieux maitrisé par les internes de médecine générale de troisième année par rapport aux internes de première année. Notre travail met en évidence un manque important déclaré de connaissances et d'habiletés des internes dans le domaine de la contraception. Conclusion : Ce travail préliminaire permet de considérer des objectifs pédagogiques qui devront être planifiés dans le cadre d'un programme dédié à l'enseignement de la santé sexuelle.

Abstract - Background: Contraception is one of the major fields in the applied studies degree (DES) program for post-graduate interns in general medicine in France. Objective: Our goal was first to collect information on reported theoretical knowledge and practical skills related to contraception from medical interns enrolled in the DES program and secondly to determine their training needs. Methods: In order to determine the knowledge and skills, the medical
\end{abstract}




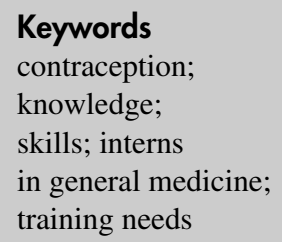

interns enrolled in the DES program were asked to fill out an Internet questionnaire. The assessment of items was determined out using the expert panel method. Results: None of the 17 theoretical-knowledge and practical-skill items tested displayed significantly better results among third-year students in general medicine compared to first-year students. The work underscores the interns' substantial lack of knowledge and know-how in the area of contraception. Conclusion: Our study should be considered as a preliminary step in determining pedagogical objectives that need to be set in the framework of a program focused on teaching sexual health.

\section{Introduction}

Les femmes françaises déclarent consulter un médecin généraliste pour le suivi et la prévention plus souvent que les hommes ${ }^{[1]} \mathrm{Ce}$ suivi comporte des soins spécifiques dits de gynécologie et d'obstétrique. Une femme consulte en moyenne en médecine générale 3,6 fois par an pour ce motif et cette fréquence augmente avec l'âge de la patiente ${ }^{[2]}$.

Aujourd'hui, en France, la prise en charge des pathologies spécifiques de gynécologie et d'obstétrique revient à quatre acteurs principaux : le gynécologue, le gynécologue obstétricien, le médecin généraliste $^{[2]}$ et plus rarement les sages-femmes. La contraception représente une partie importante de ce suivi. Les femmes utilisent dans $55 \%$ des cas la pilule, dans $25 \%$ des cas le dispositif intra utérin (DIU), dans $5 \%$ des cas l'implant, l'anneau ou le patch ${ }^{[3]}$.

Selon la World Organization of National colleges, Academies and Academic Associations of General Practitioners/Family Physicians (WONCA), le médecin généraliste doit être capable d' «assurer le suivi et la continuité des soins lors des problèmes et pathologies les plus fréquentes chez les enfants et adolescents, les femmes, les hommes, les personnes âgées " ${ }^{[4]}$. Dans son référentiel de métiers et compétences, le collège des généralistes enseignants intègre la contraception comme une des compétences du métier de généraliste ${ }^{[5]}$.

Une formation adéquate est nécessaire car lors des entretiens relatifs à la prescription d'un contraceptif, le professionnel doit composer avec des éléments médicaux et psychosociaux. Ces facteurs peuvent avoir des conséquences sur l'adhésion des patientes à la contraception proposée et rendre difficile l'observance ou la bonne utilisation de la méthode ${ }^{[6]}$.
En 2010, l'enquête FECOND, réalisée par l'Institut national de prévention et d'éducation pour la santé (INPES) et l'Institut national des études démographiques (INED), a examiné les pratiques récentes en matière de contraception, au regard des méthodes utilisées selon l'âge des femmes et leur milieu social et des pratiques des professionnels de santé ; les auteurs soulignaient que les professionnels de santé ont des difficultés à adapter leurs pratiques et que leur formation en matière de contraception est un « enjeu essentiel ${ }^{[3]}$.

Ainsi, dans le cadre d'une démarche d'évaluation de programme, il nous a semblé important de réaliser une analyse descriptive et critique de l'état de certaines connaissances et savoir-faire déclarés des internes du diplôme d'études spécialisées (DES) de médecine générale de la faculté de médecine de Poitiers, concernant la contraception, cette évaluation nous permettant de déterminer, pour partie, si les conditions actuelles de formation concernant la contraception sont adéquates au regard des recommandations actuelles.

\section{Méthodes}

La population étudiée était celle des internes de médecine générale de la subdivision d'internat de Poitou-Charentes au cours du semestre mainovembre 2011, soit 210 étudiants.

Pour recueillir les informations, nous avons élaboré un questionnaire de 17 items, administré anonymement en ligne aux étudiants via le site internet de la faculté de médecine de Poitiers. Les internes étaient invités à préciser leur semestre 
d'internat (Annexe 1). Les non-répondeurs ont été relancés trois fois par mail.

Les items utilisés pour le questionnaire ont été déterminés par six experts recrutés selon la méthode dite « boule de neige » ${ }^{[7]}$. Les experts devaient exercer la médecine générale et avoir une expertise en santé sexuelle. Il s'agissait de deux maîtres de conférences associés, l'un d'entre eux étant chargé de cours en santé sexuelle et praticien attaché du service de gynécologie du centre hospitalier universitaire de Poitiers, de deux enseignants ambulatoires avec une activité quotidienne importante de gynécologie, les deux autres étant médecins généralistes non enseignants avec un exercice ambulatoire important en gynécologie. L'élaboration et la validation du contenu des items se sont inspirées d'une démarche de consensus formalisé, variante de la méthode de groupe nominal, développée principalement par la Rand Corporation et l'Université de Californie à Los Angeles (procédure dite Rand/Ucla), employée par la Haute autorité de santé (HAS) pour valider des recommandations cliniques ${ }^{[8]}$. Quatre phases se sont succédé : élaboration d'un contenu provisoire des différents items par les deux maîtres de conférences associés, analyse de ce contenu par les autres experts, analyse des items rajoutés ou des désaccords, rédaction du consensus finalisé. Plusieurs tours ont été nécessaires pour obtenir $70 \%$ d'accord des experts sur les items. Les items ont été formulés sous la forme de questions à réponse binaire, de questions à choix multiples, et d'une question ouverte. Un prétest a été effectué avant l'administration du questionnaire pour vérifier qu'il était compréhensible par la population cible. Dans le cadre de ce travail, le coefficient alpha de Cronbach a été utilisé comme indicateur de la fidélité des échelles de mesure. Cet indice statistique, variant entre 0 et 1 , permet d'évaluer l'homogénéité (ou cohérence interne) d'un instrument d'évaluation ou de mesure composé par un ensemble d'items qui, tous, devraient contribuer à appréhender une même entité «sous-jacente». En l'occurrence, il s'agissait du niveau de connaissances et d'habiletés déclarées des internes de médecine générale en Poitou-Charentes durant le semestre mai-novembre 2011.

\section{Résultats}

\section{Caractéristiques et qualités psychométriques du questionnaire}

Le questionnaire utilisé comprend trois parties distinctes : a) des questions à choix multiples pour lesquelles le coefficient alpha de Cronbach est incalculable ; b) des questions à réponses binaires (oui/non) mesurant l'expérimentation des internes en gynécologie de soins primaires, pour laquelle le coefficient alpha standardisé est estimé à 0,753 ; c) des questions à réponses utilisant une échelle de Likert exprimant différents degrés d'accord et mesurant l'aisance des internes face à diverses situations (êtes-vous à l'aise... ?) pour laquelle le coefficient alpha standardisé est de 0,836 .

\section{Échantillon des participants}

Après exclusion des réponses trop tardives et incomplètes, le taux de réponse a été de 52,4\% (110/210), avec une répartition équitable entre les trois années du troisième cycle des études médicales (TCEM) : 35 réponses pour les TCEM1; 34 réponses pour les TCEM 2 et 41 pour les TCEM 3. Parmi les internes ayant répondu, $61,3 \%$ avaient réalisé un stage hospitalier d'interne en gynécologie et $61,3 \%$ avaient réalisé un stage en médecine générale de niveau 1.

Données recueillies et comparaison des résultats selon l'année d'internat

L'ensemble des réponses des internes au questionnaire est répertorié dans le tableau I.

D'une manière générale, les internes déclarent presque tous avoir déjà instauré une contraception orale (88,6\% des TCEM 1 et 97,6\% des TCEM3) et 63,0\% d'entre eux disent être à l'aise pour le faire.

Dans le domaine des connaissances théoriques, plus de $90 \%$ des internes ne savent pas que l'acronyme BERCER (Bienvenue-Entretien-Renseignement-Choix-Explication-Retour) désigne une modalité de prescription de la contraception, alors qu'ils 
Tableau I. Progression des connaissances et des savoir-faire déclarés par les internes de médecine générale au cours du troisième cycle des études médicales (TCEM).

\begin{tabular}{|c|c|c|c|c|c|c|c|c|c|c|}
\hline & \multirow[b]{3}{*}{$n=$} & \multicolumn{3}{|c|}{ Annies de TCEM } & \multicolumn{3}{|c|}{ Comparaison TCEM1/TCEM3 } & \multicolumn{3}{|c|}{ Nombre de sujets par année } \\
\hline & & \multirow{3}{*}{\begin{tabular}{|c|} 
1ere \\
35 \\
\end{tabular}} & \multirow{2}{*}{$\frac{2 \mathrm{e} m e}{34}$} & \multirow{2}{*}{$\frac{3 \operatorname{sime}}{41}$} & \multirow{2}{*}{ Che } & \multirow{3}{*}{$\rho$} & \multirow{2}{*}{ test } & \multirow{2}{*}{$\frac{1 \text { ere }}{35}$} & \multirow{2}{*}{$\frac{2 \mathrm{emme}}{34}$} & \multirow{2}{*}{$\frac{3 e m e}{41}$} \\
\hline & & & & & & & & & & \\
\hline \multicolumn{9}{|l|}{ Savez-vous faire? } & & \\
\hline prescrire une $\mathrm{CO}^{\prime}$ & & $88,6 \%$ & $82,4 \%$ & $97,6 \%$ & & 0.174 & Fisher & 31 & 28 & 40 \\
\hline changer une $\mathrm{CO}^{\prime}$ & & $62,9 \%$ & $61.8 \%$ & $85,4 \%$ & 5,100 & $0.024^{*}$ & कोi & 22 & 21 & 35 \\
\hline prescrie un quick start & & $8.6 \%$ & $2.9 \%$ & $9,8 \%$ & 0,048 & 0.826 & yates & 3 & 1 & 4 \\
\hline prescrire la $\mathrm{CU}^{2}$ & & $34,3 \%$ & $29,4 \%$ & $36,6 \%$ & 0,04 & 0.835 & $\mathrm{ch}^{2}$ & 12 & 10 & 15 \\
\hline donner les consignes si oublic & $\mathrm{SCO}^{\prime}$ & $65,7 \%$ & $70,6 \%$ & $85.4 \%$ & 4.03 & $0.045^{*}$ & chir & 23 & 24 & 35 \\
\hline \multicolumn{11}{|l|}{ Etes-vous a raise pour? } \\
\hline preschire une $\mathrm{CO}^{\prime}$ & & $62,9 \%$ & $04,7 \%$ & $63,4 \%$ & 0,00 & 0,959 & कhi & 22 & 22 & 26 \\
\hline changer une $\mathrm{CO}^{+}$ & & $40,0 \%$ & $58.8 \%$ & $61,0 \%$ & 3,33 & 0,068 & chi p $^{2}$ & 14 & 20 & 25 \\
\hline \multicolumn{11}{|l|}{ Pensez-vous connaitro? } \\
\hline \multirow[t]{3}{*}{ Consignes si oubli de CO' } & oui & $31.4 \%$ & $41,2 \%$ & $41,5 \%$ & 0,82 & 0,366 & $\mathrm{ch}^{2}$ & 11 & 14 & 17 \\
\hline & pas certain $(\theta)$ & $51,4 \%$ & $50.0 \%$ & $48,8 \%$ & & & & 18 & 17 & 20 \\
\hline & mon & $17,1 \%$ & $8.8 \%$ & $9.8 \%$ & & & & 6 & 3 & 4 \\
\hline \multicolumn{11}{|l|}{ Test de connaissances : } \\
\hline \multirow[t]{3}{*}{ Conditions du quick start } & connait & $14,3 \%$ & $8.8 \%$ & $19.5 \%$ & 0,36 & 0,546 & chir & 5 & 3 & 8 \\
\hline & ne connalt pas & $82,9 \%$ & $79,4 \%$ & $63,4 \%$ & 3.57 & $0,059^{*}$ & ohit & 29 & 27 & 26 \\
\hline & & $5.7 \%$ & $2,9 \%$ & $17,1 \%$ & 1,37 & 0.241 & yates & 2 & 1 & 7 \\
\hline \multirow{3}{*}{$\begin{array}{l}\text { si oubli de } \mathrm{CO} \text { ' dans les } 7 \\
\text { demiers jours de la plaquette }\end{array}$} & ne pas fair la pause de 7 jours & $48.6 \%$ & $58,8 \%$ & $56,1 \%$ & 0,43 & 0,512 & $\mathrm{chi}^{2}$ & 17 & 20 & 23 \\
\hline & $C U^{2}$ si rapport les Si proctionts & $77,1 \%$ & $50,0 \%$ & $58,5 \%$ & 2,96 & 0,085 & $\mathrm{ch}^{2}$ & 27 & 17 & 24 \\
\hline & comnait les 2 consignes & $37,1 \%$ & $32,4 \%$ & $26,8 \%$ & 0,36 & 0,546 & $\mathrm{chi}^{2}$ & 13 & 11 & 11 \\
\hline \multicolumn{11}{|l|}{ Le DIU": } \\
\hline \multirow{2}{*}{\multicolumn{2}{|c|}{ est une contraception d"urgence }} & $60,0 \%$ & $38,2 \%$ & $63,4 \%$ & 0,09 & 0,79 & $c h R^{2}$ & 21 & 13 & 26 \\
\hline est possible sinulliparitét & & $85,7 \%$ & $94,3 \%$ & $97,6 \%$ & & 0,09 & fisher & 30 & 33 & 40 \\
\hline \multicolumn{11}{|l|}{ Savoir-faire: $\quad f=$} \\
\hline \multirow{2}{*}{\multicolumn{2}{|c|}{$\begin{array}{l}\text { poser Nexplanon } \\
\text { retirer Nexplanon }\end{array}$}} & $54,3 \%$ & $35.3 \%$ & $61.0 \%$ & 0,35 & 0.556 & chi & 19 & 12 & 25 \\
\hline & & $54,3 \%$ & $32,4 \%$ & $51,2 \%$ & 0,07 & 0,790 & chip & 19 & 11 & 21 \\
\hline \multirow{2}{*}{\multicolumn{2}{|c|}{ poser DiU" }} & $42,9 x$ & $23,5 \%$ & $36,6 \%$ & 0,31 & 0,58 & $c h i^{2}$ & 15 & 8 & 15 \\
\hline & & $54,3 \%$ & $41,2 \%$ & $46,3 \%$ & 0,48 & 0,49 & $\mathrm{ch}^{2}$ & 19 & 14 & 19 \\
\hline
\end{tabular}

'CO = contraception orale; ${ }^{2} \mathrm{CU}$ = contraception d'urgence; ${ }^{3} \mathrm{OIU}=$ dispositif intra-utérin

TCEM 1 : première année du troisième cycle des études médicales.

TCEM 3 : troisième année du troisième cycle des études médicales.

Test du $\mathrm{Chi}^{2}$ et Correction de Yates, si besoin. Test exact de Fisher en cas d'effectif théorique inférieur à 3 .

ont quasiment tous déjà été confrontés à une demande de prescription ou de changement de méthode contraceptive.

Par ailleurs, ils sont davantage à se déclarer plus à l'aise concernant le changement de contraception en fin de cursus, mais cette différence n'est pas significative $(61,0 \%$ en TCEM $3 v s .40 \%$ en TCEM1, $p=0,07$ ).

Respectivement $65 \%$ et $85 \%$ des internes de TCEM1 et de TCEM3 ont déjà pris en charge un oubli de pilule mais seuls respectivement $31 \%$ et $41 \%$ d'entre eux pensent connaître les consignes à donner dans cette situation.
Concernant l'oubli de prise de contraception orale relativement aux sept derniers comprimés de la plaquette, environ la moitié des internes de TCEM 1 et de TCEM 3 déclarent savoir qu'il ne faut pas faire la pause de sept jours $(48,6 \%$ et $56,1 \%$ respectivement, $p=0,51)$, tandis que les internes de TCEM 1 sont plus nombreux que les TCEM 3 à savoir qu'il faut prendre la contraception d'urgence s'il y a eu un rapport dans les cinq jours précédant l'oubli, différence cependant non significative (77\% vs. $58 \%$; $p=0,09$ ).

Respectivement $14,3 \%$ et $19,5 \%$ des étudiants de TCEM 1 et de TCEM $3(p=0,83)$ disent connaître 


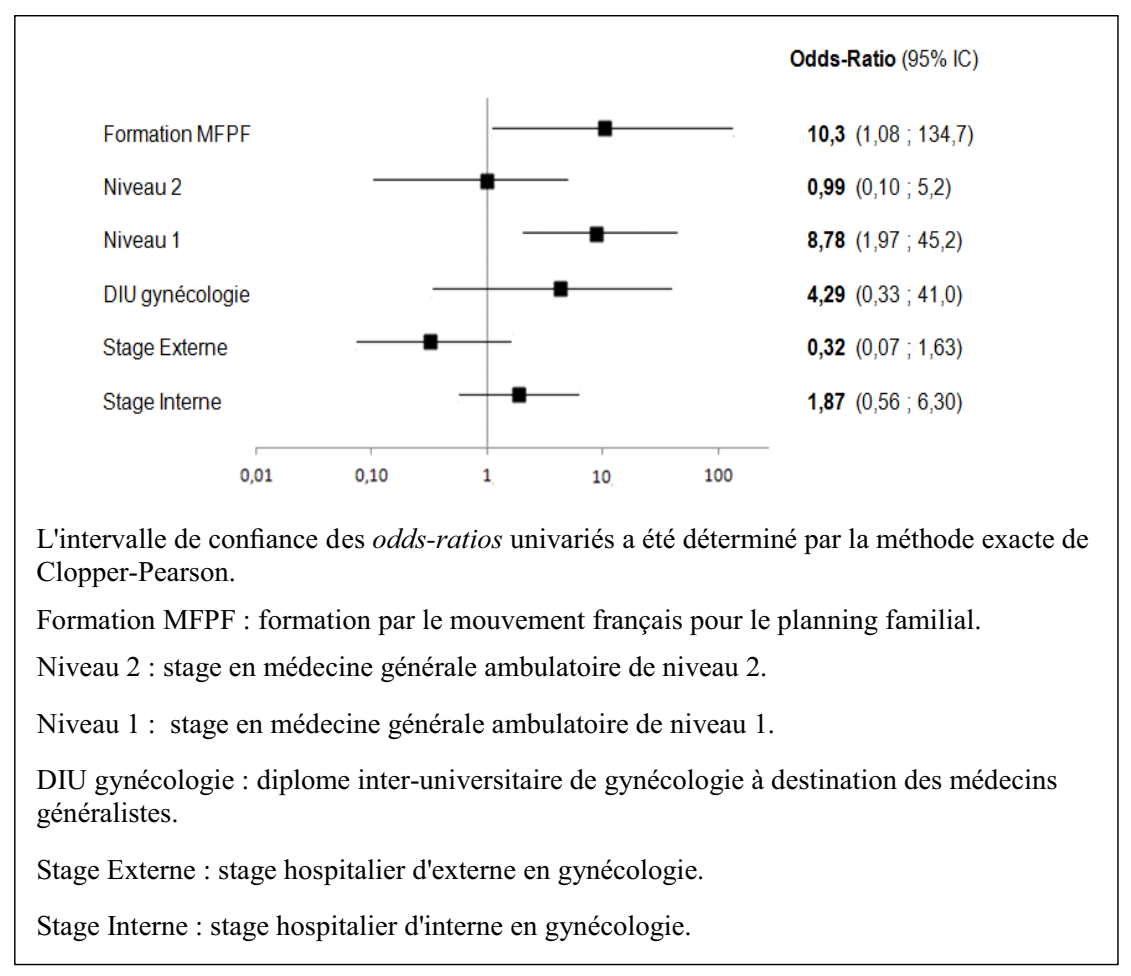

Fig. 1. Impact des différentes formations sur l'aisance ressentie pour prescrire la contraception orale.

la procédure dite «quick start» qui consiste à débuter la prise de pilule en dehors du premier jour des règles, c'est-à-dire quel que soit le moment du cycle. Trente-cinq pourcent d'entre eux en moyenne, indépendamment du semestre, ont déjà prescrit la contraception d'urgence.

Cinquante et un pour cent des internes déclarent savoir poser un implant contraceptif à l'étonogestrel et $46,1 \%$ déclarent savoir le retirer ; 35,6\% d'entre eux disent savoir poser un DIU.

\section{Évaluation de chaque mode de formation}

Il existe une corrélation significative entre le fait d'avoir suivi une formation proposée par le Mouvement français pour le planning familial (MFPF) ou d'avoir effectué le stage ambulatoire de médecine générale de niveau 1 et l'aisance ressentie pour prescrire la contraception orale (odds-ratios significativement supérieurs à 1 , figure 1 ).

\section{Discussion}

À travers l'analyse des 110 questionnaires reçus, notre étude révèle des déficits de connaissances et d'habiletés déclarées des internes de médecine générale, qui devraient être pris en compte en tant que besoins de formation relativement à la contraception de longue durée.

\section{Forces et limites de l'étude}

Les techniques de recueil d'informations utilisables dans le cadre d'un processus d'identification des 
besoins de formation sont nombreuses : questionnaires écrits, groupes de discussion focalisée, entrevues individuelles, évaluation des pratiques professionnelles, test de connaissances ${ }^{[9]}$. Nous avons choisi d'administrer un questionnaire, méthode simple à mettre en place, peu coûteuse et d'exploitation facile. En revanche, cette option méthodologique comporte des limites. Bien que les énoncés du questionnaire aient été élaborés à l'issue d'une démarche méthodique et systématique de recherche de consensus d'experts (procédure de Rand/Ucla), ce qui confère une certaine garantie quant à sa validité, la validité de construit n'a pas été formellement vérifiée. Il existait un biais de sélection dans le recrutement de ces experts car ils étaient tous issus de la faculté de médecine de Poitiers ; ainsi nous ne pouvons éliminer une similitude des pratiques, ce qui peut affecter la pertinence du choix des items retenus. En revanche, la dimension de consistance interne de la fidélité a été établie grâce au calcul d'un coefficient de Cronbach supérieur à 0,75 .

Il s'agit par ailleurs d'une collecte d'information non interactive et réductrice, qui repose sur une autoévaluation des internes, qui est, par conséquent, limitée à la validité d'un système déclaratif. Dans le cas présent cependant, nous pouvons penser que le questionnaire reflète assez bien la réalité concernant l'état de leurs connaissances et habiletés, en raison du taux de participation à 52,4\%. Cependant, il convient de ne pas négliger l'éventuelle existence d'un déficit dans l'auto-évaluation de la part de certains étudiants ou au contraire une surestimation de leurs connaissances réelles par d'autres.

Parmi les 17 items proposés, trois portaient sur des procédures dénommées par un acronyme (BERCER) ou par une métaphore énoncée en anglais (quick start). Il est probable que certains étudiants maîtrisaient effectivement ces méthodes, tout en ignorant leurs fondements théoriques et leurs désignations. Pourtant le «quick start» est une méthode en soi connue maintenant depuis 11 ans ${ }^{[10]}$. La méthode BERCER ${ }^{[11]}$ est par ailleurs décrite par plusieurs organismes tels que l'INPES, l'HAS ${ }^{[12]}$ et l'Organisation mondiale de la santé, qui sont des ressources facilement accessibles pour les étudiants. La méconnaissance de ce qu'est la méthode BERCER pourrait traduire un manque de connaissance des nouvelles recommandations de bonnes pratiques, visant à délivrer des conseils sur la contraception dans un processus de communication bi-directionnelle entre le praticien et le patient. Mais ce point reste à éclaircir car d'autres techniques existent et sont référencées. Nous souhaitions savoir si les éléments les plus récents qui participent à la prescription de cette contraception étaient connus comme tels. Ainsi, les réponses à ces questions étaient difficilement interprétables. Malgré cela, nous avons choisi délibérément de ne pas interroger les internes sur les contre-indications à la prescription de la contraception car il nous semblait important d'aller au-delà des questions habituellement posées ${ }^{[13]}$.

Les scores recueillis respectivement pour les 17 items de connaissances et de savoir-faire n'étaient pas différents chez les internes de TCEM3 et chez ceux de TCEM1. Cependant, un biais est lié au fait que l'enquête ne comparait pas les internes entrant en TCEM et ceux qui en sortaient, mais les internes respectivement en milieu de semestre 2 et de semestre 6 . Dans la mesure où la formation dure au total six semestres, les résultats des internes de TCEM 3 reflétaient donc bien les connaissances acquises en fin de cursus mais ceux des TCEM 1 ne reflétaient pas exactement les connaissances à l'entrée en TCEM. La progression entre le début et la fin du TCEM a donc pu être sous-évaluée.

Par ailleurs, il est possible que plusieurs comparaisons auraient atteint la significativité statistique si l'échantillon avait été plus important, le faible nombre de réponses limitant la puissance de l'étude.

\section{Signification des résultats}

Ce travail met en évidence un manque important de connaissances et d'habiletés déclarées des internes dans le domaine de la contraception, alors que le médecin généraliste prescrit la contraception d'une femme sur deux ${ }^{[14]}$ et que la Société française de médecine générale la place au septième rang des résultats de consultations les plus fréquents des femmes en médecine générale ${ }^{[15]}$. 
Concernant la pose de DIU et de l'implant contraceptif, les internes de médecine générale apparaissaient insuffisamment formés puisque plus de $50 \%$ d'entre eux ne les ont jamais réalisés, et que seuls 35,6\% d'entre eux déclarent savoir poser un DIU, alors qu'il s'agit du deuxième moyen de contraception le plus utilisé en France (17,3\%), après la pilule contraceptive $(45,4 \%)$. Nos chiffres sont en cohérence avec l'étude FECOND 2012 qui révèle que $29 \%$ seulement des médecins généralistes se disent préparés à la pose de stérilet à l'issue de leur cursus ${ }^{[3]}$. La HAS recommande pourtant une meilleure connaissance de son utilisation et des bonnes pratiques de sa pose, en raison de son efficacité et de son faible taux d'utilisation chez les jeunes femmes ${ }^{[16]}$. La maîtrise de la pose de ces dispositifs est d'autant plus importante que ce sont des moyens de contraception dont la prescription augmente. En effet, entre les années 2012 et 2013, la vente des contraceptifs de longue durée d'action (implant au levonorgestrel et DIU) a augmenté de $26 \%{ }^{[17]}$.

L'analyse de l'aisance ressentie pour la prescription de la contraception en fonction du lieu de stage suggère que les stages dans les services de gynécologie améliorent peu l'aisance ressentie pour la prescription de la contraception. Notre étude ne révèle pas les causes potentielles de ce ressenti. On peut néanmoins, du fait des retours des internes sur l'évaluation de ces lieux de stage (données non publiées), évoquer deux causes : la difficulté d'assister aux consultations et la moindre importance accordée à la prise en charge holistique. Un précédent travail ${ }^{[15]}$ avait mis en évidence que la participation à la consultation reste le poste pédagogique le plus efficace, correspondant, au mieux, au futur mode d'exercice de l'interne.

Les étudiants qui se déclarent être le plus à l'aise en matière de contraception sont ceux ayant effectué le stage en médecine générale ambulatoire de niveau 1 et/ou la formation avec le MFPF. Ces formations ont en commun d'être au plus près des préoccupations du médecin généraliste. Cela paraît évident pour le stage en médecine générale ambulatoire de niveau 1 puisqu'il se déroule au sein d'un cabinet de médecine générale. Le MFPF est un organisme privé agréé par l'état comme organisme de formation.
À Poitiers, la formation avec le MFPF résulte d'une planification pédagogique centrée sur les compétences professionnelles à acquérir. Les outils pédagogiques utilisés et l'expérience des formateurs favorisent l'acquisition d'un savoir-être et d'un savoir-faire nouveau. Les étudiants qui choisissent de s'y inscrire ont probablement un intérêt plus important pour les questions de soins de la femme, ce qui peut expliquer de meilleurs résultats au questionnaire.

\section{Conclusions et perspectives}

Les dispositifs de formation du troisième cycle de médecine générale relèvent de l'autonomie pédagogique des facultés de médecine et de leurs départements de médecine générale. Ainsi, nos résultats ne sont pas généralisables à l'ensemble des internes de médecine général en France. Ce travail confirme, comme d'autres avant, que l'enseignement de la contraception n'est que partiellement satisfaisant. Pourtant, la formation des médecins généralistes en contraception est un enjeu important pour le système de santé. Elle doit permettre d'assurer un accès équitable aux dépistages et aux soins.

Ce travail plaide pour le développement des lieux de stages visant l'enseignement des soins spécifiques concernant la femme, qu'il s'agisse de stages en médecine générale, de stages en collaboration avec des structures telles que le MFPF ou, à défaut, de dispositifs recourant à la simulation sur mannequins.

\section{Contributions des auteurs}

Stéphanie Grandcolin a dirigé le travail, élaboré la méthodologie et participé à la rédaction du manuscrit. Jean Du breuilliac a effectuée l'analyse statistique et participé à la rédaction du manuscrit. AnneLaure Chapuis a participé à la réalisation de l'étude et à la rédaction du manuscrit. François Birault et José Gomes ont participé à la relecture du manuscrit.

\section{Approbation éthique}

Non sollicitée 


\section{Déclaration d'intérêts}

SG déclare n'avoir aucun conflit d'intérêts, JDB déclare n'avoir aucun conflit d'intérêts, FB déclare n'avoir aucun conflit d'intérêts, JG déclare n'avoir aucun conflit d'intérêts, A-LC déclare n'avoir aucun conflit d'intérêts.

\section{Références}

1. Danet S, Olier L. La santé des femmes en France. Paris : La documentation française, 2009.

2. Cohen J, Madelenat P, Levy-Toledano R. Gynécologie et santé des femmes, quel avenir en France ? Paris : Eska, 2000.

3. Bajos N, Bohet A, Le Guen M, Moreau C. L'équipe de l'enquête Fecond. Population \& Sociétés 2012 (492). [On-line] Disponible sur : http://www.ined.fr/ fichier/t_telechargement/49903/ telechargement_fichier_fr_publi_pdf1_492.pdf

4. WONCA EUROPE. Les définitions européennes des caractéristiques de la discipline de médecine générale, du rôle du médecin généraliste et une description des compétences fondamentales du médecin généraliste - médecin de famille. 2002 [On-line] Disponible sur : http://www.cnge.fr.

5. Collectif : Collège national des généralistes enseignants et Mission nationale d'évaluation des compétences professionnelles des métiers de la santé Référentiel métiers et compétences: médecins généralistes, sages-femmes et gynécologues obstétriciens. Paris : Berger-Levrault, 2010.

6. Institut national de prévention et d'éducation pour la santé (INPES). Contraception : les françaises utilisent-elles un contraceptif adapté à leur mode de vie ? 2011 [On-line] Disponible sur: http:// www.inpes.sante.fr/70000/dp/11/dp111026.pdf.

7. Letrillart L, Bourgeois I, Vega A, Cittée J, Lutsman M. Un glossaire d'initiation à la recherche qualitative. Première partie : d' « Acteur » à «Interdépendance ». Exercer 2009;20:74-9.

8. Haute autorité de santé (HAS). Bases méthodologiques pour l'élaboration de recommandations professionnelles par consensus formalisé. 2006 [On-line] Disponible sur: http://www.hassante.fr/portail/upload/docs/application/pdf/ base_methodo_CFE.pdf.

9. Honorat C, Levasseur G. Quels besoins enseigner, quel besoin d'enseigner? Pédagogie Médicale 2001; 2:26-30.

10. Westhoff C, Kerns J, Morroni C, Cushman LF, Tiezzi L, Murphy PA. Quick start: novel oral contraceptive initiation method. Contraception. 2002;66:141-5.

11. Rinehart W, Rudy S, Drennan M. Gather guide to counseling. Popul Rep J 1998(48):1-31.

12. Haute autorité de santé (HAS). Fiche mémo. Contraception: prescriptions et conseils aux femmes. 2013 [On-line] Disponible sur : http://www.has-sante.fr/portail/upload/docs/ application/pdf/2013-03/fiche_contraception_ conditions_prescription.pdf.

13. Gelly M. Avortement et contraception dans les études médicales. Une formation inadaptée. Paris : L'Harmattan, 2006.

14. Collectif. Le médecin généraliste et suivi de la femme - Enquête Epilule. 2008 [On-line] Disponible sur : http://www.mgfrance.org/

15. Société française de médecine générale. Observatoire de médecine générale. Classement des 50 résultats de consultations les plus fréquents par patients pour les femmes pour l'année 2009. 2009 [On-line] Disponible sur : http://omg.sfmg.org.

16. Institut national de prévention et d'éducation pour la santé (INPES). Stratégies de choix des méthodes contraceptives chez la femme. 2004 [On-line] Disponible sur: http://www.inpes.sante.fr/ 70000/dp/04/dp041207.pdf.

17. Agence nationale de sécurité des médicaments et des produits de santé (ANSM). Evolution de l'utilisation en France des contraceptifs oraux combinés et autres contraceptifs de décembre 2012 à août 2013. 2013 [On-line] Disponible sur : http://ansm.sante.fr/

Correspondance et offprints : Stéphanie Grandcolin, 37 place des templiers, 86000 Poitiers, France.

Mailto : stephaniegrandcolin@aliceadsl.fr 